\title{
CLOSED-FORM FREQUENCY ESTIMATION USING SECOND-ORDER NOTCH FILTERS
}

\author{
S.M. Savaresi ${ }^{\dagger}$, S. Bittanti ${ }^{\dagger}$, H.C. So* \\ ${ }^{\dagger}$ Dipartimento di Elettronica e Informazione, Politecnico di Milano, Piazza L. da Vinci, \\ 32, 20133 Milano, ITALY. \\ *Department of Computer Eng. and Information Tech., City University of Hong Kong, \\ Tat Chee Avenue, HONG KONG.
}

\begin{abstract}
In this paper the problem of the frequency estimation of a sinusoid embedded in white noise is considered. The approach used herein is the minimization of the sample variance of the output of constrained notch filters fed by the noisy sinusoid. In particular, this paper focuses on closed-form expressions of the frequency estimate, which can be obtained using notch filters having an all-zeros FIR structure. In this paper it is shown that the FIR notch filters obtained from standard $2^{\text {nd }}$-order IIR filters are inadequate, and an alternate $2^{\text {nd }}$-order IIR notch filter is proposed, which provides an unbiased estimate of the frequency. The FIR filter obtained from the new IIR filter provides a closed-form unbiased frequency estimate. Copyright (C) 2003 IFAC
\end{abstract}

Keywords. Frequency estimation; harmonic analysis; notch filters; unbiased parameter identification.

\section{INTRODUCTION}

This paper deals with the estimation of the frequency of a harmonic signal $s(t)=A \cos \left(\Omega_{0} t+\varphi\right)$, given its noisy measurement $y(t)=s(t)+n(t), t=1,2, \ldots N$, where $n(t)$ is a zero-mean white Gaussian noise $\left(n \sim W G N\left(0, \sigma^{2}\right)\right)$. This problem is frequently encountered in real-world applications, especially in the fields of adaptive control and signal processing, and numerous techniques have been developed for its treatment (see e.g. Bittanti and Picci, 1996, Bittanti and Savaresi, 2000, Hsu et al., 1999, Kay, 1988, La Scala and Bitmead, 1996, Quinn and Fernandes, 1991, Renders et al., 1984, Savaresi et al., 2001, Schoukens et al., 1992, Stoica, 1992). This paper focuses on the class of estimation methods based on constrained notch filters (see e.g. Händel and Nehorai, 1994, and references cited therein).

The basic idea underlying notch-filters-based estimation techniques is the minimization, with respect to $\Omega$, of the loss function

$$
J(\Omega)=\sum_{t=1}^{N} \varepsilon(t, \Omega)^{2},
$$

where $\varepsilon(t, \Omega)=G\left(z^{-1}, \Omega\right) y(t)$ is the output of a notch filter with transfer function $G\left(z^{-1}, \Omega\right)$, fed by the measured signal $y(t)$. The notch of $G\left(z^{-1}, \Omega\right)$ is centered around the frequency $\Omega$.

In general, the dependence of $J(\Omega)$ on $\Omega$ is nonlinear and non-convex; hence, iterative quasi-Newton minimization methods must be used. If the unknown frequency $\Omega_{0}$ is time-varying, and the minimization of (1) is made recursively, the estimation algorithm usually is called frequency tracker (see e.g. Boashash, 1992).

Obviously, the most crucial design choice in a notchbased estimation technique is the selection of the structure and of the parameterization of the filter 
$G\left(z^{-1}, \Omega\right)$. Usually, $2^{\text {nd }}$-order IIR filter with a strongly constrained parameterization are used. Starting from $2^{\text {nd }}$-order filters, simple FIR filters or more sophisticated higher-order IIR filters have been developed and proposed (Händel et al., 1998, Savaresi, 1997).

Two slightly different $2^{\text {nd }}$-order IIR notch filters are typically used in practice. They have the following expressions:

$$
\begin{aligned}
& G_{1}\left(z^{-1}, \Omega, \rho\right)=\frac{1-2 \cos (\Omega) z^{-1}+z^{-2}}{1-2 \rho \cos (\Omega) z^{-1}+\rho^{2} z^{-2}}, \\
& G_{2}\left(z^{-1}, \Omega, \rho\right)=\frac{1-2 \cos (\Omega) z^{-1}+z^{-2}}{1-\left(1+\rho^{2}\right) \cos (\Omega) z^{-1}+\rho^{2} z^{-2}} .
\end{aligned}
$$

In (2) and (3) the parameter $\rho(0 \leq \rho<1)$ is known as the de-biasing parameter or the poles-contraction factor (note that $\rho$ only affects the position of the poles). The difference of the poles position (when $\rho$ varies) of (2) and (3) can be easily appreciated from Fig.1.
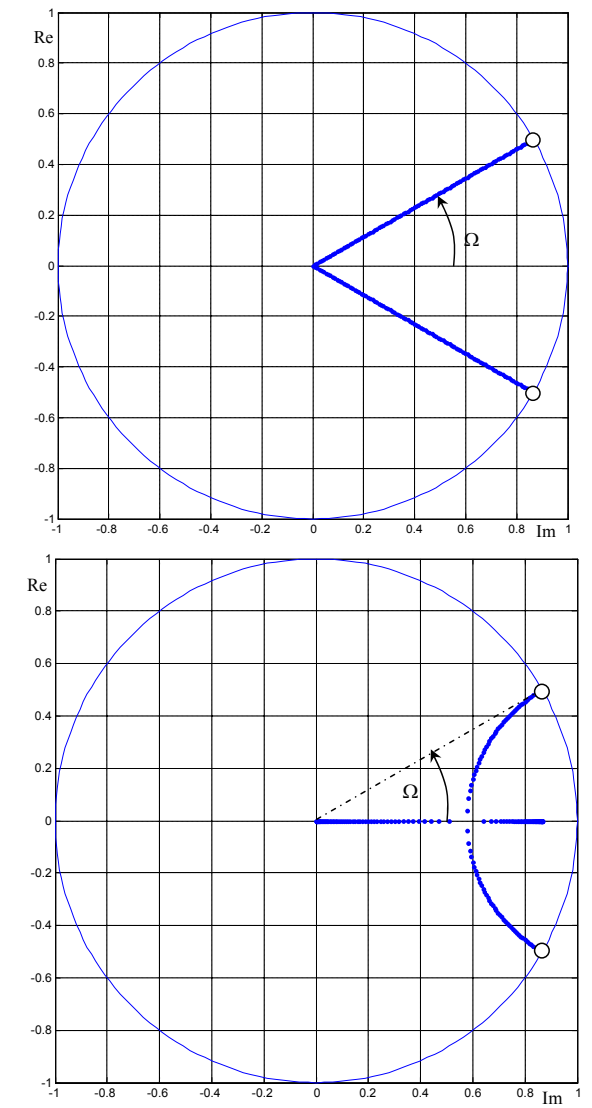

Fig.1. Example of pole placement of filters (2) (left) and (3) (right). The white bullets indicate the position of the zeros.

In the literature, filters of this type are also known as constrained notch filter, where the term constrained refers to the fact that their structure is strongly underparameterized: the 5 parameters of a fullyparameterized $2^{\text {nd }}$-order digital IIR filter are reduced to one parameter only. As a matter of fact, since $\rho$ is regarded as a design parameter, the only unknown parameter of (2) and (3) is the angular frequency $\Omega$. The main difference between (2) and (3) is that (3) provides a rigorously unbiased estimation of the frequency of a pure tone embedded in white noise, whereas (2) provides a biased estimate. It is easy to see that such bias is negligible if $\rho \approx 1$; the problem of the bias becomes severe if $\rho<<1$. The properties of such filters have been discussed and analyzed in a large number of works (see e.g. Bittanti et al., 1997, and references cited therein).

The goal of this paper is to develop closed-form frequency estimators based on notch filters. The starting point of this work can be summarized in the following observations:

- closed-form expressions of the frequency estimator cannot be obtained if the notch filter has a IIR structure, due to the auto-regressive part of the filter;

- a constrained FIR notch filter can be obtained from $G_{1}\left(z^{-1}, \Omega, \rho\right)$ by setting $\rho=0$; this is not possible using $G_{2}\left(z^{-1}, \Omega, \rho\right.$ ) (note that $G_{2}\left(z^{-1}, \Omega, 0\right)$ is not a FIR);

- the closed-form frequency estimate obtained from $G_{1}\left(z^{-1}, \Omega, 0\right)$ is severely affected by a bias error.

Starting from these observations, the main results and original contributions of this paper are the following: a new $2^{\text {nd }}$-order IIR unbiasing constrained notch filter $G_{3}\left(z^{-1}, \Omega, \rho\right)$ is developed and analyzed (section 2); it is shown, in Section 3, that a closed-form frequency estimate can be obtained using the FIR filters $G_{1}\left(z^{-1}, \Omega, 0\right)$ and $G_{3}\left(z^{-1}, \Omega, 0\right)$ (the major advantage of $G_{3}\left(z^{-1}, \Omega, 0\right)$ over $G_{1}\left(z^{-1}, \Omega, 0\right)$ is that it provides a rigorously unbiased estimate of $\Omega_{0}$ ); it is shown that the closed-form frequency estimate provided by the FIR notch filter $G_{3}\left(z^{-1}, \Omega, 0\right)$, if the number $N$ of data snapshots is large, tends to the frequency estimators provided by the "Pisarenko Harmonic Decomposition" approach, and by the "Youle-Walker" approach (Section 4).

\section{A NEW UNBIASING NOTCH FILTER}

As already remarked in the Introduction, one of the major drawbacks of the notch filter (2) (the most widely used in practice) is that it provides a biased estimation of $\Omega_{0}$. This bias is particularly severe when $\rho<<1$. Starting from the cost function (1), a new unbiasing $2^{\text {nd }}$-order IIR notch filter can be obtained as follows.

Consider the long-run (asymptotic) version of the cost function (1), namely:

$$
\bar{J}(\Omega)=\lim _{N \rightarrow \infty} \frac{1}{N}\left(\sum_{t=1}^{N} \varepsilon(t, \Omega)^{2}\right),
$$

where $\varepsilon(t, \Omega)=G\left(z^{-1}, \Omega\right) y(t)$.

It is easy to see that $\bar{J}(\Omega)$ can be given the following 
expression (see Bittanti et al., 1997):

$\bar{J}(\Omega)=\frac{1}{2 \pi} \int_{-\pi}^{+\pi}\left|G\left(e^{j \omega}, \Omega\right)\right|^{2} S_{y}(\omega) d \omega$,

where $S_{y}(\omega)$ is the power spectrum of $y(t)$, which can be split into the power spectra of $s(t)$ and $n(t)$, namely: $S_{y}(\omega)=S_{s}(\omega)+S_{n}(\omega) ; \quad S_{n}(\omega)=\sigma^{2}$ and $S_{s}(\omega)=\frac{A^{2}}{2}\left(\frac{1}{2} \delta\left(\omega+\Omega_{0}\right)+\frac{1}{2} \delta\left(\omega-\Omega_{0}\right)\right)$.

Compute the asymptotic cost function $\bar{J}_{1}(\Omega)$ associated with the notch filter $G_{1}\left(z^{-1}, \Omega, \rho\right)$, by plugging in (4) the expression of the notch filter (2) and the expressions of $S_{s}(\omega)$ and $S_{n}(\omega)$ :

$$
\begin{aligned}
& \bar{J}_{1}(\Omega)=\bar{J}_{1}^{(s)}(\Omega)+\bar{J}_{1}^{(n)}(\Omega) ; \\
& \bar{J}_{1}^{(n)}(\Omega)=\frac{1}{2 \pi} \int_{-\pi}^{+\pi}\left|G_{1}\left(e^{j \omega}, \Omega, \rho\right)\right|^{2} \sigma^{2} d \omega, \\
& \bar{J}_{1}^{(s)}(\Omega)=\frac{1}{2 \pi}\left(\frac{A^{2}}{2} G_{1}\left(e^{j \omega}, \Omega_{0}, \rho\right)\right) .
\end{aligned}
$$

For the computation of $\bar{J}_{1}^{(n)}(\Omega)$ (the contribution to $\bar{J}_{1}(\Omega)$ due to the noise) we have resorted to the Rugizka algorithm (see Åström, 1970). The calculus of $\bar{J}_{1}^{(s)}(\Omega)$ calls for cumbersome but easier computations. The expressions obtained for $\bar{J}_{1}^{(s)}(\Omega)$ and $\bar{J}_{1}^{(n)}(\Omega)$ are:

$$
\begin{aligned}
\bar{J}_{1}^{(s)}(\Omega) & =\frac{2 A^{2}\left(\cos (\Omega)-\cos \left(\Omega_{0}\right)\right)^{2}}{D E N} \\
D E N= & 1+\rho^{4}+4 \rho^{2} \cos ^{2}\left(\Omega_{0}\right)+ \\
& +4 \rho^{2} \cos ^{2}(\Omega)-4 \rho^{3} \cos (\Omega) \cos \left(\Omega_{0}\right)+ \\
& -4 \rho \cos (\Omega) \cos \left(\Omega_{0}\right)-2 \rho^{2} \\
\bar{J}_{1}^{(n)}(\Omega)= & \frac{\sigma^{2}}{\pi} \frac{\rho^{3}+\rho^{2}-6 \rho \cos ^{2}(\Omega)+\rho+2 \cos ^{2}(\Omega)+1}{(1+\rho)\left(\rho^{2}+2 \rho \cos (\Omega)+1\right)\left(\rho^{2}-2 \rho \cos (\Omega)+1\right)} .
\end{aligned}
$$

The bias in the frequency estimate obtained using $G_{1}\left(z^{-1}, \Omega, \rho\right)$ is due to the fact that $\bar{J}_{1}^{(n)}(\Omega)$ is a function of $\Omega$. This dependence of $\bar{J}_{1}^{(n)}(\Omega)$ on $\Omega$ has the effect of moving the minimum of $\bar{J}_{1}(\Omega)$ away from $\Omega_{0}$ (whereas $\Omega_{0}$ is the minimum of $\bar{J}_{1}^{(s)}(\Omega)$ ). Now observe that the minimum of $\bar{J}_{1}^{(s)}(\Omega)$ does not change if $\bar{J}_{1}^{(s)}(\Omega)$ is multiplied by a strictly positive function of $\Omega$ and $\rho$, say $\eta(\Omega, \rho$ ) (obviously for $\Omega \in[0, \pi)$ and $\rho \in[0,1))$. This is due by the presence of the factor $\left(\cos (\Omega)-\cos \left(\Omega_{0}\right)\right)$ in $\bar{J}_{1}^{(s)}(\Omega)$, which is null if $\Omega=\Omega_{0}$. Consider then the following function $\eta(\Omega, \rho)$ :

$$
\eta(\Omega \rho)=\sqrt{\frac{(1+\rho)\left(\rho^{2}+2 \rho \cos (\Omega)+1\right)\left(\rho^{2}-2 \rho \cos (\Omega)+1\right)}{\left(1+\rho^{2}\right)\left(\rho^{3}+\rho^{2}-6 \rho \cos ^{2}(\Omega)+\rho+1+2 \cos ^{2}(\Omega)\right)}}(6)
$$

Note that such function is the square-root of the inverse of $\bar{J}_{1}^{(n)}(\Omega)$ (but for the coefficient $\sigma^{2} / \pi$ ), multiplied by $\left.\left(1+\rho^{2}\right)\right)$.

A new unbiasing filter $G_{3}\left(z^{-1}, \Omega, \rho\right)$ can be obtained from $G_{1}\left(z^{-1}, \Omega, \rho\right)$ and (6) as follows:

$$
\begin{aligned}
& G_{3}\left(z^{-1}, \Omega, \rho\right)=\eta(\Omega, \rho) G_{1}\left(z^{-1}, \Omega, \rho\right)= \\
& =\eta(\Omega, \rho) \frac{1-2 \cos (\Omega) z^{-1}+z^{-2}}{1-2 \rho \cos (\Omega) z^{-1}+\rho^{2} z^{-2}}
\end{aligned}
$$

Due to the fact that $G_{3}\left(z^{-1}, \Omega, \rho\right)$ is simply obtained by multiplying $G_{1}\left(z^{-1}, \Omega, \rho\right)$ by $\eta(\Omega, \rho)$, some remarks on the shape of $\eta(\Omega, \rho)$ are due (see Fig.2 where $\eta(\Omega, \rho)$ is plotted in the ranges $\Omega \in[0, \pi]$ and $\rho \in[0,1])$.

- $\quad \eta(\Omega, \rho)$ is not-null in the ranges $\Omega \in[0, \pi]$ and $\rho \in[0,1]$; this can be easily seen from (6). This guarantees the well-posedness of the optimization problem based on the cost function (1).

- Note that $\eta(\Omega, 1)=1$, whereas $\eta(\Omega, 0)$ strongly differs from 1 ; this is expected since $\eta(\Omega, \rho)$ is a sort of "de-biasing factor" of $G_{1}\left(z^{-1}, \Omega, \rho\right)$. Therefore $\eta(\Omega, \rho)$ leaves $G_{1}\left(z^{-1}, \Omega, \rho\right)$ almost unchanged if $\rho$ is close to 1 , whereas $\eta(\Omega, \rho)$ provides a strong correction to $G_{1}\left(z^{-1}, \Omega, \rho\right)$ for small values of $\rho$.

- Note that $\eta(\pi / 2, \rho)=1 \quad \forall \rho \in[0,1]$, and that $\eta(\Omega, \rho)$ is symmetric with respect to $\Omega=\pi / 2$ in the range $\Omega \in[0, \pi]$ (Bittanti et al., 1997).

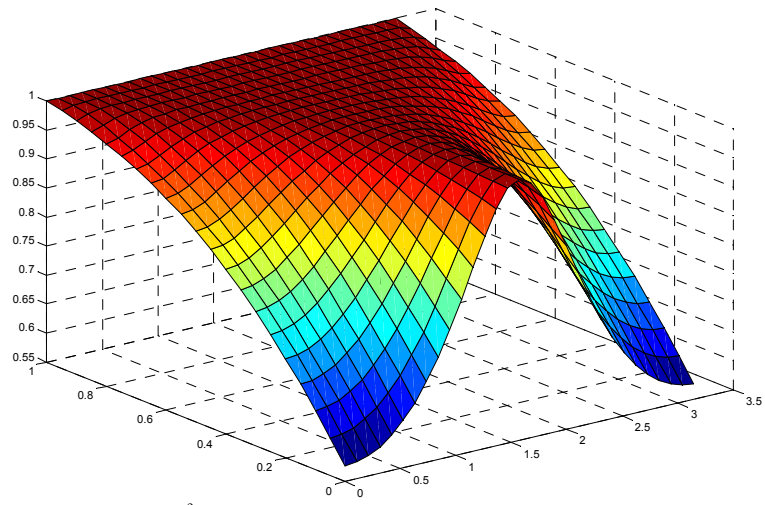

Fig.2. Shape of the function $\eta(\Omega, \rho)$ in the ranges

$$
\Omega \in[0, \pi] \text { and } \rho \in[0,1] \text {. }
$$

In order to get a complete understanding of the differences between the three $2^{\text {nd }}$-order constrained notch filters $G_{1}\left(z^{-1}, \Omega, \rho\right), G_{2}\left(z^{-1}, \Omega, \rho\right)$, and $G_{3}\left(z^{-1}, \Omega, \rho\right)$, it is interesting to compare the corresponding asymptotic cost functions $\bar{J}_{1}(\Omega)$, $\bar{J}_{2}(\Omega)$, and $\bar{J}_{3}(\Omega)$, respectively. 
The closed-form expressions of $\bar{J}_{1}(\Omega)$ has already been computed in (5). Following the same procedure, $\bar{J}_{2}(\Omega)=\bar{J}_{2}^{(s)}(\Omega)+\bar{J}_{2}^{(n)}(\Omega)$ and $\bar{J}_{3}(\Omega)=\bar{J}_{3}^{(s)}(\Omega)+\bar{J}_{3}^{(n)}(\Omega)$ can be obtained as:

$$
\begin{aligned}
\bar{J}_{2}^{(s)}(\Omega)= & \frac{2 A^{2}\left(\cos (\Omega)-\cos \left(\Omega_{0}\right)\right)^{2}}{D E N} \\
D E N= & +\rho^{4} \cos ^{2}(\Omega)-2 \rho^{4} \cos (\Omega) \cos \left(\Omega_{0}\right)-2 \rho^{2}+ \\
& +\rho^{4}+\cos ^{2}(\Omega)+2 \rho^{2} \cos ^{2}(\Omega)-4 \rho^{2} \cos (\Omega) \cos \left(\Omega_{0}\right)+ \\
& -2 \cos (\Omega) \cos \left(\Omega_{0}\right)+4 \rho^{2} \cos ^{2}\left(\Omega_{0}\right) \\
\bar{J}_{2}^{(n)}(\Omega) & =\frac{\sigma^{2}}{\left(1+\rho^{2}\right) \pi} \\
\bar{J}_{3}^{(s)}(\Omega) & =\frac{2 A^{2}\left(\cos (\Omega)-\cos \left(\Omega_{0}\right)\right)^{2} \eta^{2}(\Omega \rho)}{D E N} \\
D E N= & +\rho^{4}+4 \rho^{2} \cos ^{2}\left(\Omega_{0}\right)+4 \rho^{2} \cos ^{2}(\Omega)+ \\
& -4 \rho^{3} \cos (\Omega) \cos \left(\Omega_{0}\right)-4 \rho \cos (\Omega) \cos \left(\Omega_{0}\right)-2 \rho^{2} \\
\bar{J}_{3}^{(n)}(\Omega) & =\frac{\sigma^{2}}{\left(1+\rho^{2}\right) \pi} .
\end{aligned}
$$

By comparing $\bar{J}_{1}(\Omega), \bar{J}_{2}(\Omega)$, and $\bar{J}_{3}(\Omega)$ it is apparent that $\bar{J}_{2}(\Omega)$ and $\bar{J}_{3}(\Omega)$ have the minimum exactly at $\Omega_{0}$ (unbiased estimate), since $\bar{J}_{2}^{(n)}(\Omega)$ and $\bar{J}_{3}^{(n)}(\Omega)$ do not depend on $\Omega$. On the contrary, as already observed, $\bar{J}_{1}(\Omega)$ provides a biased estimate since $\bar{J}_{1}^{(n)}(\Omega)$ is $\Omega$-dependent.

To conclude this section, it is worth remarking that the new filter $G_{3}\left(z^{-1}, \Omega, \rho\right)$ merges the two main appealing features of $G_{1}\left(z^{-1}, \Omega, \rho\right) \quad$ and $G_{2}\left(z^{-1}, \Omega, \rho\right)$ :

- similarly to $G_{1}\left(z^{-1}, \Omega, \rho\right)$, a FIR filter can be obtained from $G_{3}\left(z^{-1}, \Omega, \rho\right)$ by using $\rho=0$;

- similarly to $G_{2}\left(z^{-1}, \Omega, \rho\right), \quad G_{3}\left(z^{-1}, \Omega, \rho\right)$ provides an unbiased estimate of $\Omega_{0}$ $\forall \rho \in[0,1)$.

These features will be fully exploited in the following section, in order to obtain closed-form frequency estimates based on FIR notch filters.

\section{CLOSED-FORM FREQUENCY ESTIMATION}

A closed-form notch-based frequency estimate cannot be obtained if the filter has a IIR structure. Consider the FIR filters obtained by simply setting $\rho=0$ in (2) and in (7) (it has been already observed that setting $\rho=0$ in $G_{2}\left(z^{-1}, \Omega, \rho\right)$ does not yield a FIR filter), namely:

$$
\begin{aligned}
& G_{1}\left(z^{-1}, \Omega, 0\right)=1-2 \cos (\Omega) z^{-1}+z^{-2}, \\
& G_{3}\left(z^{-1}, \Omega, 0\right)=\sqrt{\frac{1}{2 \cos ^{2}(\Omega)+1}}\left(1-2 \cos (\Omega) z^{-1}+z^{-2}\right) .
\end{aligned}
$$

Using such filters, closed-form frequency estimators from the data can be obtained as follows.

Closed form frequency estimator obtained using $G_{1}\left(z^{-1}, \Omega, 0\right)$.

Consider the following cost function, obtained by plugging in (1) the FIR notch filter $G_{1}\left(z^{-1}, \Omega, 0\right)$ :

$$
J_{1}(\Omega)=\sum_{t=1}^{N}(y(t)-2 \cos (\Omega) y(t-1)+y(t-2))^{2},
$$

and differentiate $J_{1}(\Omega)$ with respect to $\Omega$ :

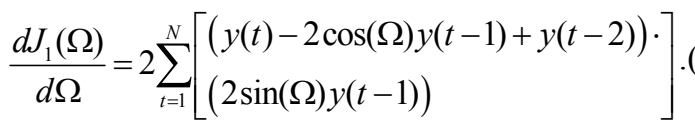

Note that (10) is quadratic with respect to $\cos (\Omega)$; hence by solving $d J_{1}(\Omega) / d \Omega=0$ with respect to $\cos (\Omega)$, it is easy to see that the following holds:

$\cos (\Omega)=\frac{\sum_{t=1}^{N} y(t-1)(y(t)+y(t-2))}{\sum_{t=1}^{N} y(t-1)^{2}}$.

The closed-form frequency estimator therefore is given by:

$\hat{\Omega}_{1}=\arccos \left(\frac{\sum_{t=1}^{N} y(t-1)(y(t)+y(t-2))}{\sum_{t=1}^{N} y(t-1)^{2}}\right)$.

As the number $N$ of data grows, $\hat{\Omega}_{1}$ tends to the minimum of the asymptotic cost function (5) (in the special case of $\rho=0$ ). After some cumbersome computation, the following asymptotic expression of (11) is obtained:

$$
\hat{\Omega}_{1}=\underset{N \rightarrow \infty}{\rightarrow} \arccos \left(\frac{\pi A^{2}}{\pi A^{2}+\sigma^{2}} \cos \left(\Omega_{0}\right)\right) \text {. }
$$

From (12), it is apparent that the frequency estimate is affected by a severe bias; note that the bias is null in the (trivial and unrealistic) case of zero noise $\left(\sigma^{2}=0\right)$; it grows as the SNR decreases.

\section{Closed form frequency estimator obtained using} $G_{3}\left(z^{-1}, \Omega, 0\right)$.

Consider the following cost function, obtained by plugging in (1) the FIR notch filter $G_{3}\left(z^{-1}, \Omega, 0\right)$ :

$$
J_{3}(\Omega)=\sum_{t=1}^{N}\left(\frac{(y(t)-2 \cos (\Omega) y(t-1)+y(t-2))^{2}}{2 \cos ^{2}(\Omega)+1}\right) \text {, }
$$

and differentiate $J_{3}(\Omega)$ with respect to $\Omega$ :

$$
\begin{aligned}
\frac{d J_{3}(\Omega)}{d \Omega}= & \sum_{t=1}^{N}\left(\frac{N U M}{(2+\cos (2 \Omega))^{2}}\right) \\
N U M= & 2(y(t)-2 \cos (\Omega) y(t-1)+y(t-2)) . \\
& \cdot\left(\begin{array}{l}
(2+\cos (2 \Omega)) 2 \sin (\Omega) y(t-1)+ \\
+\sin (2 \Omega)(y(t)-2 \cos (\Omega) y(t-1)+y(t-2))
\end{array}\right)
\end{aligned}
$$


Consider now the problem of solving $d J_{3}(\Omega) / d \Omega=0$ with respect to $\Omega$. After some manipulation the following expression is obtained:

$\sum_{t=1}^{N}\left(\begin{array}{c}\sin (\Omega)(y(t)-2 \cos (\Omega) y(t-1)+y(t-2)) \cdot \\ \cdot(y(t-1)+\cos (\Omega) y(t)+\cos (\Omega) y(t-2))\end{array}\right)=0$.

Equation (14) admits a trivial solution: $\sin (\Omega)=0$. Assuming that $\Omega_{0} \neq\{0, \pi\}$, the following quadratic form (with respect to $\cos (\Omega)$ ) can be obtained from (14):

$$
\begin{aligned}
& 2\left[\sum_{t=1}^{N} y(t-1)(y(t)+y(t-2))\right] \cos ^{2}(\Omega)+ \\
& +\left[\sum_{t=1}^{N}\left(2 y(t-1)^{2}-(y(t)+y(t-2))^{2}\right)\right] \cos (\Omega)+ \\
& -\left[\sum_{t=1}^{N} y(t-1)(y(t)+y(t-2))\right]=0
\end{aligned}
$$

From (15), a closed-form frequency estimator can be computed. It has the following expression:

$$
\begin{aligned}
& \hat{\Omega}_{3}=\arccos \left(\frac{N U M}{4\left[\sum_{t=1}^{N} y(t-1)(y(t)+y(t-2))\right]}\right) \\
& N U M=-\left[\sum_{t=1}^{N}\left(2 y(t-1)^{2}-(y(t)+y(t-2))^{2}\right)\right]+ \\
& +\sqrt{\left[\sum_{t=1}^{N}\left(2 y(t-1)^{2}-(y(t)+y(t-2))^{2}\right)\right]^{2}+8\left[\sum_{t=1}^{N} y(t-1)(y(t)+y(t-2))\right]^{2}}
\end{aligned}
$$

As the number $N$ of data grows, $\hat{\Omega}_{3}$ tends to the minimum of the asymptotic cost function (8) (in the special case of $\rho=0$ ):

$\hat{\Omega}_{3} \underset{N \rightarrow \infty}{\rightarrow} \Omega_{0}$.

Thus, the new filter $G_{3}\left(z^{-1}, \Omega, 0\right)$ provides a simple closed-form unbiased estimate of $\Omega_{0}$. Interestingly, (16) is closely related to the method given in So, 2002 and in So and Ip, 2002, even if the derivation of this result is completely different.

\section{RELATED METHODS}

In the literature, other closed-form frequency estimators for harmonic signals in white noise have been proposed and analyzed. Two celebrated estimators are the "Youle-Walker" estimator, and the "Pisarenko Harmonic Decomposition" (PHD) estimator (see e.g. Pisarenko, 1973, Xiao and Takodoro, 1994 and 1995). In this section they will be briefly recalled and compared with the asymptotic version of the notch-based estimator (16).

\section{Youle-Walker approach.}

Given $\quad y(t)=s(t)+n(t), \quad s(t)=A \cos \left(\Omega_{0} t+\varphi\right)$, $n \sim \operatorname{WGN}\left(0, \sigma^{2}\right)$, the autocorrelation coefficients of order 1 and 2, say $r_{1}$ and $r_{2}$ respectively, are given by:

$$
\left\{\begin{array}{l}
r_{1}=E[y(t) y(t-1)]=\frac{A^{2}}{2} \cos \left(\Omega_{0}\right) \\
r_{2}=E[y(t) y(t-2)]=\frac{A^{2}}{2} \cos \left(2 \Omega_{0}\right)
\end{array}\right.
$$

By eliminating the parameter $A$ in (17), the following equation is obtained:

$2 r_{1} \cos ^{2}\left(\Omega_{0}\right)-r_{2} \cos \left(\Omega_{0}\right)-r_{1}=0$.

Its solution with respect to $\Omega_{0}$ provides the YouleWalker frequency estimator, given by:

$$
\hat{\Omega}_{Y W}=\arccos \left(\frac{r_{2}+\sqrt{r_{2}^{2}+8 r_{1}^{2}}}{4 r_{1}}\right) \text {. }
$$

\section{PHD approach.}

Given a zero-mean stationary signal $y(t)$, its autocorrelation matrix of order 3 is given by:

$$
\begin{aligned}
R & =\left[\begin{array}{lll}
r_{0} & r_{1} & r_{2} \\
r_{1} & r_{0} & r_{1} \\
r_{2} & r_{1} & r_{0}
\end{array}\right], \\
r_{0} & =E\left[y(t)^{2}\right], r_{1}=E[y(t) y(t-1)], r_{2}=E[y(t) y(t-2)] .
\end{aligned}
$$

The eigenvector associated with the smallest eigenvalue of $R$ has the following form:

$$
\left[\begin{array}{cc}
1-\frac{r_{2}+\sqrt{r_{2}^{2}+8 r_{1}^{2}}}{2 r_{1}} & 1
\end{array}\right]^{T} \text {. }
$$

Pisarenko (Pisarenko, 1973) has proven that, if $y(t)=s(t)+n(t), s(t)=A \cos \left(\Omega_{0} t+\varphi\right), \quad n \sim W G N\left(0, \sigma^{2}\right)$, the smallest eigenvalue of $R$ must have the following simple expression:

$$
\left[\begin{array}{lll}
1 & -2 \cos \left(\Omega_{0}\right) & 1
\end{array}\right]^{T} \text {. }
$$

By comparing (19) and (20), the PHD frequency estimator is obtained:

$$
\hat{\Omega}_{P H D}=\arccos \left(\frac{r_{2}+\sqrt{r_{2}^{2}+8 r_{1}^{2}}}{4 r_{1}}\right) \text {. }
$$

Interestingly enough, the Youle-Walker and PHD approaches provide exactly the same results. This has been recently proven and discussed in Xiao and Takodoro, 1994 and 1995.

Consider now the notch-based closed-form estimator (16). The following result holds.

\section{Proposition 1.}

Given a signal $y(t)=s(t)+n(t)$, where $s(t)=A \cos \left(\Omega_{0} t+\varphi\right), n \sim W G N\left(0, \sigma^{2}\right)$, the notchfilter based estimator (16) asymptotically converges towards $\hat{\Omega}_{Y W}$ and $\hat{\Omega}_{P H D}$, namely:

$\lim _{N \rightarrow \infty} \hat{\Omega}_{3}=\arccos \left(\frac{r_{2}+\sqrt{r_{2}^{2}+8 r_{1}^{2}}}{4 r_{1}}\right)$, 
$r_{1}=E[y(t) y(t-1)], \quad r_{2}=E[y(t) y(t-2)]$.

Proof.

If $N$ is large, the following hold:

$$
\begin{aligned}
& \lim _{N \rightarrow \infty}\left[\frac{1}{N} \sum_{t=1}^{N} y(t-1)(y(t)+y(t-2))\right]= \\
& =\lim _{N \rightarrow \infty}\left[\frac{1}{N} \sum_{t=1}^{N} 2 y(t) y(t-1)\right]=2 E[y(t) y(t-1)] \\
& \lim _{N \rightarrow \infty}\left[\frac{1}{N} \sum_{t=1}^{N}\left(2 y(t-1)^{2}-(y(t)+y(t-2))^{2}\right)\right]= \\
& =-2 E[y(t) y(t-2)]
\end{aligned}
$$

By plugging in (16) the asymptotic expressions (22), it is easy to see that:

$$
\begin{aligned}
& \lim _{N \rightarrow \infty} \hat{\Omega}_{3}=\arccos \left(\frac{r_{2}+\sqrt{r_{2}^{2}+8 r_{1}^{2}}}{4 r_{1}}\right), \\
& r_{1}=E[y(t) y(t-1)], \quad r_{2}=E[y(t) y(t-2)] .
\end{aligned}
$$

From a theoretical point of view the fact that (asymptotically) $\hat{\Omega}_{Y W}, \hat{\Omega}_{P H D}$ and $\hat{\Omega}_{3}$ are exactly the same, is particularly interesting: it shows the equivalence of three classical approaches which have been independently conceived and developed following three completely different paths.

\section{ACKNOWLEDGMENTS}

This work has been supported by MIUR project "New Methods for Identification and Adaptive Control for Industrial Systems", and by the EU project "Nonlinear and Adaptive Control".

\section{REFERENCES}

Åström, K.J. (1970). Introduction to stochastic control theory. Academic Press.

Bittanti S., Campi M., Savaresi S.M. (1997). Unbiased estimation of a sinusoid in noise via Adapted Notch Filters. Automatica, vol.33, n.2, pp.209-215.

Bittanti, S., G. Picci (Eds.) (1996). Identification, Adaptation, Learning - The Science of Learning Models from Data. Computer and Systems Sciences Series, Springer-Verlag, Berlin.

Bittanti S., Savaresi S.M. (2000). On the parametrization and design of an Extended Kalman Filter Frequency Tracker. IEEE Transactions on Automatic Control, vol.45, n.9, pp.1718-1724.

Boashash B. (1992). Estimating and Interpreting the Instantaneous Frequency of a Signal. Proceedings of the IEEE, vol.80, n.4, pp.520-568.

Händel P., and Nehorai A. (1994). Tracking Analysis of an Adaptive Notch Filter with Constrained Poles and Zeros. IEEE Transactions on Signal Processing, vol.42, n.2, pp.281-291.

Händel P., Tichavsky P., Savaresi S.M. (1998). Large error recovery for a class of frequency tracking algorithms. International Journal on Adaptive Control and Signal Processing, Vol.12, pp.417436.

Hsu L., Ortega R., Damm G. (1999). A Globally Convergent Frequency Estimator. IEEE Transactions on Automatic Control, Vol.44, No.4, pp.698-713.

Kay S.M. (1988). Modern Spectral Estimation: Theory and Applications. Prentice-Hall.

La Scala B., Bitmead R. (1996). Design of an Extended Kalman Filter Frequency Tracker. IEEE Transactions on Signal Processing, Vol.44, n.3, pp.739-742.

Quinn B.G, Fernandes J.M. (1991). A Fast Efficient Technique for the Estimation of Frequency. Biometrika, Vol.78, pp.489-497.

Pisarenko V.F. (1973). The retrieval of harmonics from a covariance function. J.Roy.Astr., Vol.33, pp.374-376.

Renders H., Schoukens J., Vilain G. (1984). HighAccuracy Spectrum Analysis of Sampled Discrete Frequency Signals by Analytical Leakage Compensation. IEEE Trans. on Instrum. and Meas., vol.33, n.4, pp.287-292.

Savaresi S.M. (1997). Funnel Filters: a new class of filters for frequency estimation of harmonic signals. Automatica, Vol.33, n.9, pp.1711-1718.

Savaresi S.M., R. Bitmead, W. Dunstan (2001). Nonlinear system identification using closed-loop data with no external excitation: the case of a lean combustion process. International Journal of Control, vol.74, n.18, pp.1796-1806.

Schoukens J., Pintelon R., Van hamme H. (1992). The Interpolated Fast Fourier Transform : A Comparative Study. IEEE Trans. on Instrum. and Meas., vol.41, n.2, pp.226-232.

So H.C. (2002). "A closed form frequency estimator for a noisy sinusoid". Proceedings of 45th IEEE Midwest Symposium on Circuits and Systems, Tulsa, Oklahoma, USA.

So H.C., Ip S.K. (2002). A novel frequency estimator and its comparative performances for short record lengths. Proceedings of 11th European Signal Processing Conference, Toulouse, France.

Stoica P. (1992). List of references on spectral line analysis. Signal Processing, vol.31, pp.329-340.

Xiao Y., Tadokoro Y. (1994). On Pisarenko and constrained Yule-Walker estimators of tone frequency. IEICE Transactions, vol.E77-A, n.8, pp. 1404-1406.

Xiao Y., Tadokoro Y. (1995). Statistical analysis of a simple constrained high-order Yule-Walker tone frequency estimator. IEICE Transactions, vol.E78-A, n.10, pp. 1415-1418. 